\title{
Determinación de los gases contaminantes O2, CO2, CO, NOX y SO2 en fuentes fijas en la provincia de Cotopaxi.
}

\author{
Determination of the gaseous pollutants $\mathrm{O} 2, \mathrm{CO} 2, \mathrm{CO}, \mathrm{NOX}$ and $\mathrm{SO} 2$ in \\ stationary sources in the province of Cotopaxi.
}

Oscar Rene Daza Guerra. ${ }^{1}$, Manuel Patricio Clavijo Cevallos. ${ }^{2}$ Cristian Javier Lozano Hernández. ${ }^{3}$ \& Carlos Mantilla Parra ${ }^{4}$.

Recibido: 13-07-2017 / Revisado: 07-09-2018 Aceptado: 05-10-2018/ Publicado: 28-11-2018

\begin{abstract}
.
DOI: $\underline{\text { https://doi.org/10.33262/cienciadigital.v2i4.2..199 }}$
\end{abstract}

La presente investigación tuvo como finalidad identificar la contaminación atmosférica generada por la empresa de Cereales la Pradera y la Institución Pública del Hospital General en el Cantón Latacunga. Para la determinación de la concentración de los gases contaminantes se realizó mediciones constantes con el Analizador de Gases de Combustión TESTO 350 que evaluó los gases $\mathrm{O}_{2}, \mathrm{CO}_{2}, \mathrm{CO}$, $\mathrm{NOx}, \mathrm{SO}_{2}$, según la normativa internacional (USEPA) United States Environmental Protection Agency donde se rigen los protocolos de monitoreo, y de evaluación establecidos en TULSMA, Libro VI, Anexo 3. La investigación se realizó durante un año y se dividió en dos partes para cumplir con los objetivos planteados; en la primera parte se realizó un diagnóstico de las áreas de estudios, que incluyeron entrevistas a los jefes de mantenimiento para conocer los problemas ambientales actuales que se evidencio en los monitoreos en la segunda parte, con la información recopilada en campo se obtuvo los siguientes resultados: NOx $89,325 \mathrm{mg} / \mathrm{Nm}^{3}$ y $\mathrm{SO}_{2} \quad 12,002$ mg/Nm3 en el Hospital General de Latacunga y en la Empresa de Cereales La Pradera: NOx 121,636 mg/ $\mathrm{Nm}^{3}, \mathrm{SO}_{2} 13,442 \mathrm{mg} / \mathrm{Nm}^{3}$, NOx $120,957 \mathrm{mg} / \mathrm{Nm}^{3}$ y $\mathrm{SO}_{2}$ $44,563 \mathrm{mg} / \mathrm{Nm} 3$ en sus calderos 1 y 2 respectivamente los mismos que fueron

1 Universidad Técnica de Cotopaxi, Cotopaxi, Ecuador, oscar.daza@utc.edu.ec

2 Universidad Técnica de Cotopaxi, Cotopaxi, Ecuador, manuel.clavijo@utc.edu.ec

${ }_{3}^{3}$ Universidad Técnica de Cotopaxi, Cotopaxi, Ecuador, cristian.lozano@utc.edu.ec

${ }^{4}$ Universidad Técnica de Cotopaxi, Cotopaxi, Ecuador, carlos.mantilla@utc.edu.ec 
comparados con la normativa ambiental vigente, mediante el análisis gráfico se evidencio que existe variaciones mínimas durante los días monitoreados. Los datos obtenidos en el monitoreo atmosférico en el Hospital General de Latacunga y Empresa Cereales la Pradera genera información para la toma de decisiones de políticas ambientales a nivel empresarial.

Keywords: Calidad del Aire, Fuentes Fijas de Combustión, Calderos, Equipo Analizador de Gases de Combustión Testo 350, Límites Permisibles.

\section{Resumen.}

The possible atmospheric pollution generated by Cereales la Pradera Company and the Public Institution of the General Hospital in Latacunga Canton. For the determination the pollution gases concentration was the purpose of this research, constant measurements were made with the TESTO 350 Combustion Gas Analyzer that evaluated the $\mathrm{O}_{2}, \mathrm{CO}_{2}, \mathrm{CO}, \mathrm{NOx}, \mathrm{SO}_{2}$ gases, according to the international (USEPA) United States Environmental Protection Agency where the monitoring and evaluation protocols established in TULSMA, Book VI, Annex 3, are governed. This research was conducted during a year and was divided into two parts to meet the objectives set; in the first part a diagnosis of the study areas was carried out, which included interviews to the maintenance managers to know the current environmental problems that were evidenced in the monitoring and in the second part, with the information collected in the field was obtained the following results: NOx 89.325 $\mathrm{mg} / \mathrm{Nm} 3$ and SO $212.002 \mathrm{mg} / \mathrm{Nm} 3$ at the General Hospital of Latacunga and in the company of cereals the Prairie: NOx $121.636 \mathrm{mg} / \mathrm{Nm} 3, \mathrm{SO} 213.442 \mathrm{mg} / \mathrm{Nm} 3$, NOx $120.957 \mathrm{mg} / \mathrm{Nm} 3$ and SO $244.563 \mathrm{mg} / \mathrm{Nm} 3$ in their cauldrons 1 and 2 respectively the same as were compared with the environmental regulations in force by the Graphical analysis it was shown that there is minimal variations during the days monitored. The data obtained in the atmospheric monitoring at the General Hospital of Latacunga and Prairie Grain Company generates information for decision making in environmental policies at the enterprise level; these policies include constant monitoring.

Palabras Claves: Air Quality, Fixed Combustion Sources, Boilers, Testo 350 Combustion Gas Analyzer Equipment, Permissible Limits.

\section{Introducción.}

El Cantón Latacunga dentro de su sector industrial y de servicio en los últimos años ha tenido un incremento considerable. Por tal motivo la calidad del aire se ha visto afectada por los porcentajes de gases como el $\mathrm{CO}, \mathrm{CO}_{2}, \mathrm{NOx}$ y $\mathrm{SO}_{2}$, en el inventario de las emisiones que se generan en las fuentes fijas de combustión. De tal manera se ha visto importante realizar la investigación para determinar el porcentaje de los gases contaminantes que son emitidos hacia la atmosfera, producto de la combustión en las fuentes fijas (MAE, 2012). 


\section{Importancia del problema.}

\section{Metodología.}

\section{Área de Estudio .}

Para determinar las zonas de estudio se realizó una georreferenciación que permitió obtener sus ubicaciones.

- El Hospital General de Latacunga que se encuentra ubicada en la Ciudad de Latacunga en la parroquia la Matriz en la calle Hnas. Páez y Márquez de Maenza.

Tabla 1. Datos generales del Hospital General de Latacunga.

\begin{tabular}{|c|c|}
\hline COORDENADAS: & Norte: 9896417 \\
\hline ALTITUD: & $2777 \mathrm{msnm}$ \\
\hline $\begin{array}{lccc}\text { FECHA } & \text { DE } & \text { INICIO } & \text { DE } \\
\text { FUNCIONAMIENTO: } & & \\
\end{array}$ & 1983 \\
\hline DATOS DEL PROMOTOR: & Ing. Carlos Calapaqui \\
\hline $\begin{array}{l}\text { ACTIVIDAD DE LA INDUSTRIA } \\
\text { MONITOREADA : }\end{array}$ & Salud \\
\hline $\begin{array}{l}\text { NÚMERO DE EMPLEADOS DE LA } \\
\text { EMPRESA: }\end{array}$ & 470 Personas \\
\hline
\end{tabular}

Autor: Grupo de Investigación.

- La Empresa de Cereales La Pradera se encuentra ubicada en la Ciudad de Latacunga en la parroquia Belisario Quevedo.

Tabla 2 Datos generales Empresa de Cereales La Pradera

\begin{tabular}{|l|l|l|}
\hline COORDENADAS: & Este: $768957 \quad$ Norte: 9890893 \\
\hline $\begin{array}{l}\text { ALTITUD: } \\
\text { FECHA DE INICIO DE } \\
\text { FUNCIONAMIENTO: }\end{array}$ & 1985 \\
\hline $\begin{array}{l}\text { DATOS DEL PROMOTOR: } \\
\text { ACTIVIDAD DE LA INDUSTRIA }\end{array}$ & $\begin{array}{l}\text { Ing. Darwin Madrid } \\
\text { ventas@cerealeslapradera.com } \\
(03) \text { 226-6333 }\end{array}$ \\
$\begin{array}{l}\text { MONITOREADA: } \\
\text { NÚMERO DE EMPLEADOS DE LA } \\
\text { EMPRESA: }\end{array}$ & L6 Personas \\
\hline
\end{tabular}

Autor: Grupo de Investigaciòn. 


\section{Tipo de investigación.}

Investigación descriptiva.

La investigación descriptiva se consideró fundamental para conseguir detallar el entorno actual de las zonas en estudio y determinar el tipo de calderos que se utiliza en la empresa la Cereales la Pradera y el Hospital General de Latacunga.

\section{Investigación Bibliográfica}

En el presente proyecto la investigación bibliográfica se aplicó en el proceso de recolección e indagación de fuentes diferentes de información documental tales como archivos, documentos y web para su posterior análisis que sirvió de base para respaldar la fundamentación teórica.

\section{Investigación de Campo}

La investigación de campo permitió conocer las zonas de estudio donde se realizó el monitoreo para la recolección de los datos de los diferentes monitoreos ejecutados mediante la utilización del Analizador de Gases de Combustión TESTO 350.

\section{Métodos.}

\section{Método Descriptivo.}

Este método consintió en describir la situación actual de las actividades que desarrollaban las empresas, tipo de calderos, características de las chimeneas, determinando si cuentan o no con puertos de muestreo, una vez obtenido los datos permitió la interpretación de los datos obtenidos.

\section{Método Inductivo.}

Este método analítico y sintético permitió realizar los monitoreos de manera objetiva para la obtención de datos reales de las fuentes fijas de la Empresa Cereales la pradera y el Hospital General de Latacunga para su posterior comparación con la normativa ambiental vigente.

\section{Método Deductivo.}

Mediante la aplicación de este método se obtuvo conocimientos generales para el análisis de los datos obtenidos en el monitoreo de las zonas en estudio, para llegar a la comparación con la normativa ambiental vigente TULSMAS Libro VI, Anexo 3, Tabla 2: Límites Máximos Permisibles de Concentraciones de Emisión para Calderos Generadores de Vapor (mg/Nm3), por lo que fue útil para determinar la cantidad de gases contaminantes generados por la Empresa de Cereales La Pradera y el Hospital General de Latacunga. 
Técnicas de investigación.

Observación.

Mediante la técnica de observación permitió tener una visión de la realidad de los problemas existentes para la aplicación del protocolo del monitoreo y se adquirió información de las características de las fuentes fijas que sirvieron para el desarrollo de la investigación.

\section{Entrevista.}

Mediante la entrevista efectuada a los jefes de mantenimiento se obtuvo información sobre particularidades como tipo de calderos, tiempo de uso, si se realiza mantenimiento a los calderos que sirvieron para el desarrollo de las actividades de las empresas.

\section{Protocolo para el monitoreo de gases.}

\section{Monitoreo.}

Para el monitoreo de gases en fuentes fijas se aplicó la normativa técnica y administrativa establecida en el ANEXO 3 DEL LIBRO VI DEL TEXTO UNIFICADO DE LEGISLACIÓN SECUNDARIA DEL MINISTERIO DEL AMBIENTE NORMA DE EMISIONES AL AIRE DESDE FUENTES FIJAS establecida el 4 de noviembre del 2015 Edición Especial N`387- Registro Oficial. Dentro del mismo en su Tabla 9: menciona los métodos de medición, nos manifiesta que para la aplicación de los métodos de monitoreo nos debemos basar en el protocolo establecido por la USEPA (United States Enviromental Protection Agency).

\section{Protocolo de Muestreo}

La Agencia de Protección del Medio Ambiente (USEPA) se cataloga como una agencia reguladora decretada por el Congreso de EE.UU. Esta se encarga de autorizar, escribir normas y reglamentos que explican los detalles técnicos, operacionales y legales necesarios para implementar las leyes ambientales. Los reglamentos son requisitos obligatorios que se pueden aplicar a individuos, negocios, gobiernos estatales y locales, instituciones sin fines de lucro.

\section{Métodos (USEPA) a utilizar:}

Método 1: Selección del sitio de muestreo

Método 2: La ubicación de los puertos de muestreo, distancia de puntos de muestreo.

Método 5: Tiempo de muestreo

\section{a.- Hospital General de Latacunga}

- Para el número de puertos de muestreo en el Hospital General de Latacunga cuenta con un caldero de vapor mismo que tiene una chimenea con un diámetro de $0,40 \mathrm{~m}$ tomando 
en consideración dentro del TULSMA libro VI Anexo 3 se debe tener dos (2) puertos para aquellas chimeneas o conductos de diámetro menores 3,0 metros.

- La ubicación de los puertos de muestreo está a un ángulo de $90^{\circ}$, el caldero cuenta con puertos de monitoreo a una distancia corriente arriba de $2,10 \mathrm{~m}$.

Ilustración 1. Distancia para la medición del Hospital General de Latacunga

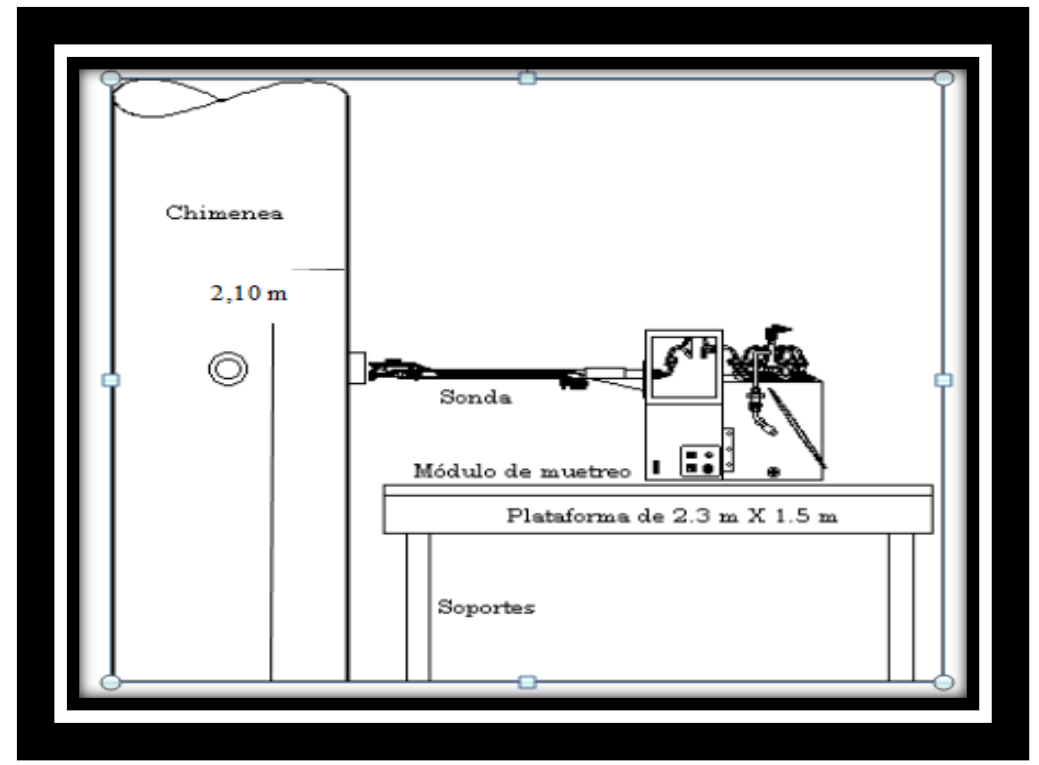

Fuente: Protocolo USEPA.

- Para la distancia de puntos de muestreo dentro de la USEPA en su Método 2, para calderas menores a 3,0 m, se debe tomar en cuenta 8 puntos de medición para el cálculo se debe tomar en consideración la Tabla 2. Distribución de puntos del monitoreo.

Tabla 3 Distribución de puntos de monitoreo.

\begin{tabular}{|l|l|l|l|l|l|l|}
\hline $\begin{array}{l}\text { Numeración del } \\
\text { punto de } \\
\text { muestreo en un } \\
\text { diámetro }\end{array}$ & 2 & 4 & 6 & 8 & 10 & 12 \\
\hline 1 & 14,6 & 6,7 & 4,4 & 3,2 & 2,6 & 2,1 \\
\hline 2 & 85,4 & 25,0 & 14,6 & 10,5 & 8,2 & 6,7 \\
\hline
\end{tabular}


Vol. 2, N4.2, p. 5-30, Octubre - Diciembre, 2018

\begin{tabular}{|c|c|c|c|c|c|}
\hline 3 & 75,0 & 29,6 & 19,4 & 14,6 & 11,8 \\
\hline 4 & 93,3 & 70,4 & 32,3 & 22,6 & 17,7 \\
\hline 5 & & 85,4 & 67,7 & 34,2 & 25,0 \\
\hline 6 & & 95,6 & 80,6 & 65,8 & 35,6 \\
\hline 7 & & & 89,5 & 77,4 & 64,4 \\
\hline 8 & & & & 85,4 & 75,0 \\
\hline 9 & & & & 91,8 & 82,3 \\
\hline 10 & & & & 97,4 & 88,2 \\
\hline 11 & & & & & 93,3 \\
\hline 12 & & & & & 97,9 \\
\hline
\end{tabular}

Fuente: Protocolo USEPA.

El cálculo de las distancias se multiplica estos valores por el diámetro de la chimenea:

Ecuación 1. Fórmula para el cálculo de la distancia de los puntos de medición.

$$
\text { Distancia }=\frac{\% \text { según los Puntos de Medición } * \text { Diametro de Chimenea }}{100}
$$

Tabla 4 Distancia de los puntos de monitoreo

\begin{tabular}{|l|l|}
\hline \multicolumn{2}{|l|}{ Hospital General de Latacunga } \\
\hline PUNTOS & DISTANCIA \\
\hline $\mathbf{1}$ & $0,0128 \mathrm{~m}$ \\
\hline $\mathbf{2}$ & $0,042 \mathrm{~m}$ \\
\hline
\end{tabular}




\begin{tabular}{|l|l|}
\hline $\mathbf{3}$ & $0,0776 \mathrm{~m}$ \\
\hline $\mathbf{4}$ & $0,1292 \mathrm{~m}$ \\
\hline $\mathbf{5}$ & $0,2708 \mathrm{~m}$ \\
\hline $\mathbf{6}$ & $0,3224 \mathrm{~m}$ \\
\hline $\mathbf{7}$ & $0,358 \mathrm{~m}$ \\
\hline $\mathbf{8}$ & $0,387 \mathrm{~m}$ \\
\hline
\end{tabular}

Fuente: Libro VI, Anexo 3 TULSMA (Mayo 2015)

\section{b.- Empresa Cereales la Pradera: Caldero 1.}

- Para el número de puertos de muestreo en la Empresa Cereales la Pradera donde cuenta con dos calderos de vapor mismos que tiene chimeneas con un diámetro de $0,3 \underline{5} \mathrm{~m}$, tomando en consideración dentro del TULSMA libro VI Anexo 3 se debe tener dos (2) puertos para aquellas chimeneas o conductos de diámetro menores 3,0 metros.

- Para la ubicación de los puertos de muestreo deben estar a un ángulo de $90^{\circ}$, en sus dos chimeneas cuenta con puertos de 2 diámetros de chimenea corriente arriba dando una distancia equivalente a $0,70 \mathrm{~m}$.

Ilustración 2. Distancias para la medición de la Empresa Cereales La Pradera

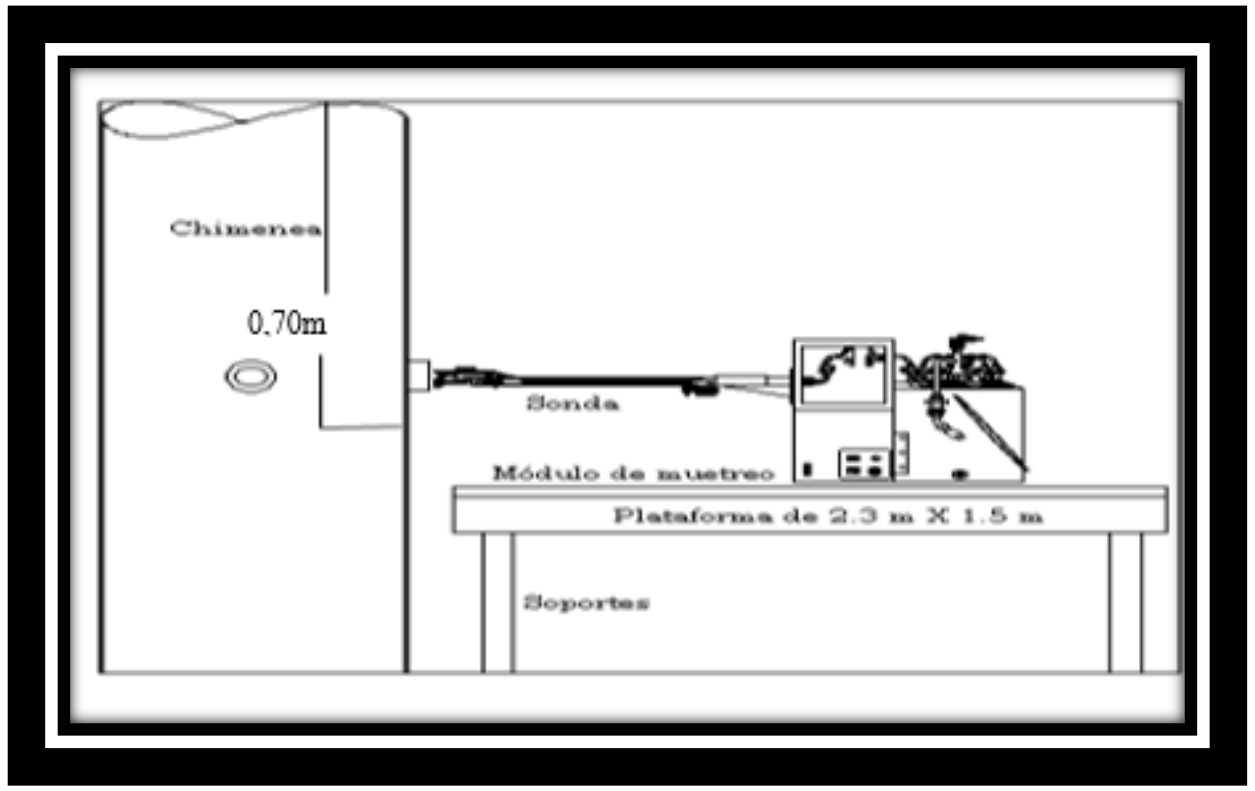

Fuente: Protocolo USEPA. 
- Para la distancia de puntos de muestreo dentro de la USEPA en su Método 2, para calderas menores a 3,0 m, se debe tomar en cuenta 8 puntos de medición para el cálculo se debe tomar en consideración la tabla 2. Distribución de puntos de los monitoreos.

Tabla 5. Distribución de puntos de monitoreo.

\begin{tabular}{|l|l|l|l|l|l|l|}
\hline $\begin{array}{l}\text { Numeración del } \\
\text { punto de muestreo } \\
\text { en un diámetro }\end{array}$ & & & & & & \\
\end{tabular}

Fuente: Protocolo USEPA.

El cálculo de las distancias se multiplica estos valores por el diámetro de la chimenea: 
Tabla 6 Distancia de los puntos de monitoreo.

\begin{tabular}{|l|l|l|}
\hline \multicolumn{2}{|l|}{ EMPRESA CEREALES LA PRADERA } \\
\hline PUNTOS & $\begin{array}{l}\text { DISTANCIA } \\
\text { CALDERO 1 }\end{array}$ & $\begin{array}{l}\text { DISTANCIA } \\
\text { CALDERO 2 }\end{array}$ \\
\hline $\mathbf{1}$ & $0,009 \mathrm{~m}$ & $0,009 \mathrm{~m}$ \\
\hline $\mathbf{2}$ & $0,031 \mathrm{~m}$ & $0,031 \mathrm{~m}$ \\
\hline $\mathbf{3}$ & $0,058 \mathrm{~m}$ & $0,058 \mathrm{~m}$ \\
\hline $\mathbf{4}$ & $0,096 \mathrm{~m}$ & $0,096 \mathrm{~m}$ \\
\hline $\mathbf{5}$ & $0,203 \mathrm{~m}$ & $0,203 \mathrm{~m}$ \\
\hline $\mathbf{6}$ & $0,242 \mathrm{~m}$ & $0,242 \mathrm{~m}$ \\
\hline $\mathbf{7}$ & $0,267 \mathrm{~m}$ & $0,267 \mathrm{~m}$ \\
\hline $\mathbf{8}$ & $0,290 \mathrm{~m}$ & $0,290 \mathrm{~m}$ \\
\hline
\end{tabular}

Fuente: Libro VI, Anexo 3 TULSMA (Mayo 2015).

\section{Tiempo de muestreo.}

Se consideró el Método 5: (USEPA).

Donde se establecen las frecuencias para realizar el monitoreo en cada punto sea continuo de los gases contaminantes como: $\mathrm{SO}_{2}$ (dióxido de Azufre), $\mathrm{NO}_{\mathrm{x}}($ Oxido Nitrico) y $\mathrm{CO}$ (Oxido de Carbono) con toma permanente durante la operación, registrando los datos máximos cada 5 minutos de los gases contaminantes, con el fin de evitar errores de la misma manera el tiempo de muestreo en cada uno de los puntos debe ser el mismo.

Resultados, Situación actual.

\section{- Hospital General de Latacunga.}

Mediante la situación actual se pudo detallar el funcionamiento de los calderos donde existe un sistema de vapor y condensado, que da servicio a las áreas de: lavandería, cocina y esterilización, en la sección de máquinas, existe dos calderos el uno se encuentra en funcionamiento, el mismos se encentran deteriorado y sin ningún tipo de mantenimiento.

La generación de vapor está a cargo del caldero de fabricación nacional EQUABOILER de $100 \mathrm{BHP}$, tiene un distribuidor de vapor y redes de aislamiento con foil de aluminio. Su funcionamiento es de12 horas al día los 8 días de la semana por ende su funcionamiento es continuo misma que generan y distribuyen el vapor a todo el sistema térmico del Hospital General de Latacunga. Debido a que la vida útil de un caldero es en promedio 20 años, la dirección del hospital está interesada en controlar y mantener el sistema de generación y distribución de vapor; que proporciona el único caldero en funcionamiento y así extender la vida útil del mismo además de garantizar el suministro de vapor a áreas vitales del Hospital General de Latacunga.

\section{- Empresa Cereales La Pradera.}


Mediante la situación actual se pudo detallar el funcionamiento de los calderos donde existe un sistema de vapor y condensado, que da servicio a las áreas de: esterilización de utensilios, en la sección de máquinas para el lavado de las mismas, existen dos calderos que se encuentran en perfecto funcionamiento, los mismos que se encuentran monitoreados constantemente.

La generación de vapor está a cargo del caldero de fabricación nacional BECKETT de 50 $\mathrm{BHP}$, tiene un distribuidor de vapor y redes de aislamiento con foil de aluminio. Su funcionamiento es de 6 horas por los 6 días a la semana de acuerdo a su producción por ende es discontinua el vapor es distribuido a todo el sistema térmico dentro de la empresa. La empresa Cereales La Pradera está interesada en controlar y mantener los parámetros principales que influyen en el sistema de generación y distribución de vapor; que proporcionan los calderos caldero en funcionamiento y así extender la vida útil del mismo además de garantizar el suministro de vapor.

\section{Resultado y análisis de los gases contaminantes identificados en las fuentes fijas.}

En la tabla se presentan los resultados de las emisiones de los gases monitoreados y su comparación con la normativa ambiental del Hospital General de Latacunga.

Tabla 7 Comparación de datos con el TULSMA Libro VI, Anexo 3 en el Hospital General de Latacunga

\section{HOSPITAL GENERAL DE LATACUNGA}

\begin{tabular}{|c|c|c|c|c|c|}
\hline GASES & UNIDADES & $\begin{array}{l}\text { PROMEDIO } \\
\text { DATOS } \\
\text { MEDIDOS }\end{array}$ & $\begin{array}{l}\text { NORMATIVA } \\
\text { ACTUAL } \\
\text { REGISTRO N } \mathbf{N}^{\circ} 387 \\
\text { DEL TULSMA } \\
4 \text { DE NOVIEMBRE } \\
\text { - } 2015\end{array}$ & $\begin{array}{l}\text { NORMATIVA } \\
\text { DEROGADA } \\
\text { REGISTRO No } \\
\text { DEL TULSMA } \\
2013\end{array}$ & $\begin{array}{l}\text { CUMPLIMIENTO } \\
\text { DE LA } \\
\text { NORMATIVA }\end{array}$ \\
\hline TH & ${ }^{\circ} \mathrm{C}$ & 145,225 & - & - & NO APLICA \\
\hline $\mathbf{O}_{2}$ & $\%$ & 13,429 & - & - & NO APLICA \\
\hline $\mathrm{CO}_{2}$ & $\%$ & 4,414 & - & - & NO APLICA \\
\hline $\mathrm{CO}$ & $\mathrm{mg} / \mathrm{Nm}^{3}$ & 12710 & - & 1800 & NO APLICA \\
\hline NOx $_{x}$ & $\mathrm{mg} / \mathrm{Nm}^{3}$ & 89,325 & 670 & - & CUMPLE \\
\hline $\mathrm{SO}_{2}$ & $\mathrm{mg} / \mathrm{Nm}^{3}$ & 12,002 & 2004 & - & CUMPLE \\
\hline
\end{tabular}

Autor: Grupo de Investigación.

\section{Interpretación:}

Desarrollado el monitoreo de gases en fuentes fijas, teniendo como característica que el caldero es de vapor del año 2013 fuente fija existente: entrar en funcionamiento con la autorización del Libro VI, Anexo 3 del TULSMA emitido en el 2003. Se obtuvo como 
resultado en el Hospital General de Latacunga lo siguientes valores: $89,325 \mathrm{mg} / \mathrm{Nm}^{3}$ de $\mathrm{NO}_{\mathrm{X}}$ (óxido de nitrógeno) y de $\mathrm{SO}_{2}$ (dióxido de azufre) $12,002 \mathrm{mg} / \mathrm{Nm}^{3}$. Los mismos que fueron comparados con los valores: $670 \mathrm{mg} / \mathrm{Nm}^{3} \mathrm{NO}_{\mathrm{X}}$ y $2004 \mathrm{mg} / \mathrm{Nm}^{3} \mathrm{SO}_{2}$ de los gases respectivamente expuestos en TABLA 2 LIMITES MÁXIMOS PERMISIBLES DE CONCENTRACIÓN DE EMISIÓN DE CONTAMINANTES AL AIRE PARA CALDERAS. De tal manera demostrando que los valores de las concentraciones de los gases se encuentran dentro de los límites máximos permisibles.

Análisis: En el Hospital General de Latacunga el parámetro Monóxido de carbono CO da como resultado: $12710 \mathrm{mg} / \mathrm{Nm}^{3}$ en la normativa ambiental actual no aplica, mientras que en la Normativa derogada TULSMA del 2013 no obedece con los límites máximos permisibles.

\section{Variación diaria del monitoreo del Óxido de Nitrógeno (NOx) del Hospital General de Latacunga}

En el presente grafico se muestra la variación, del máximo, la media y el minino de los valores de concentración del óxido de nitrógeno obtenidos durante cinco días de monitoreo.

Gráfico 1 Análisis diario del monitoreo del $\left(\mathrm{NO}_{\mathrm{X}}\right)$

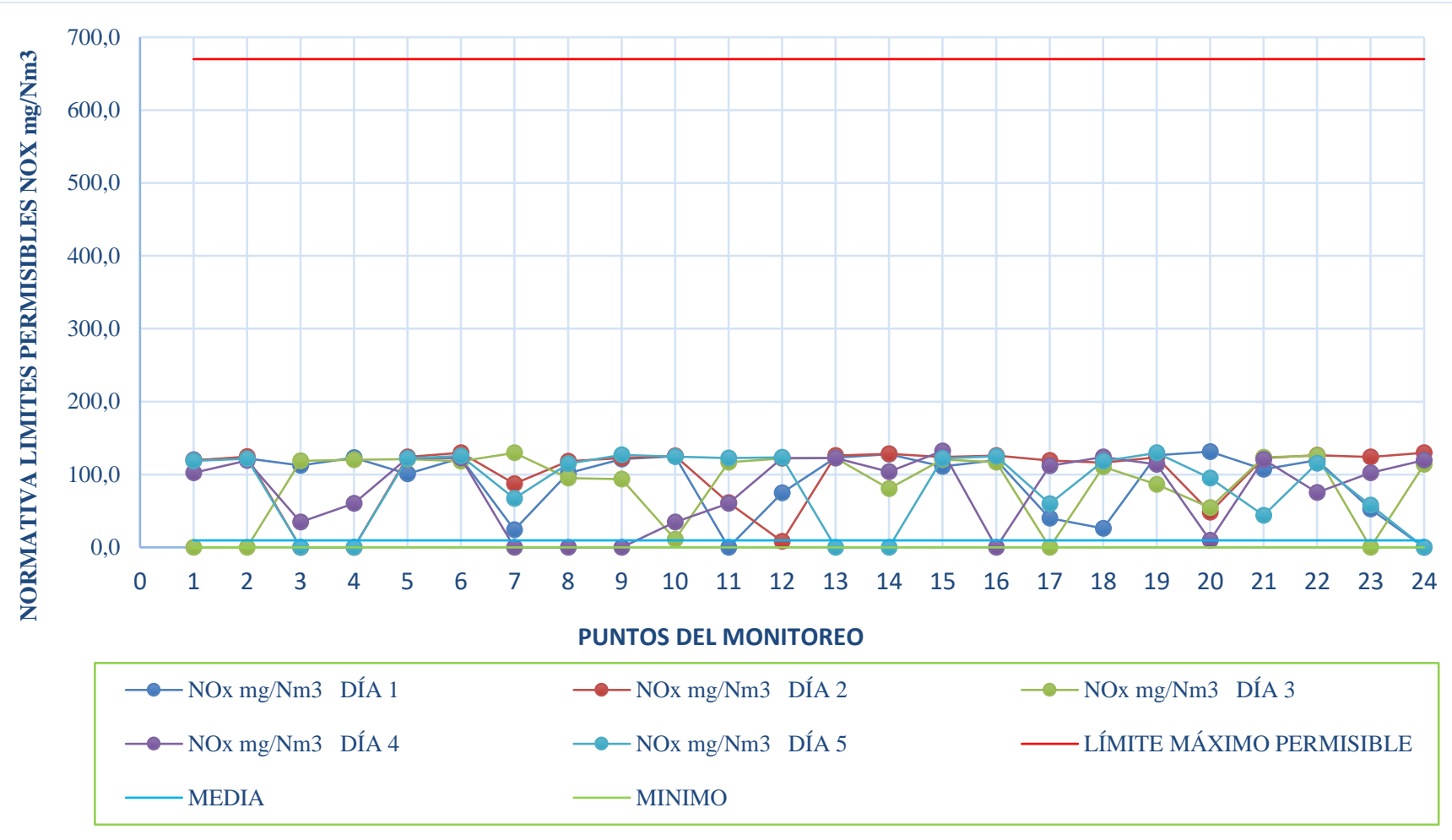

Autor: Grupo de Investigación. 


\section{Interpretación del gráfico:}

En el gráfico se puede evidenciar los resultados del monitoreo realizado durante cinco días en sus 24 puntos de medición, donde se observa que el valor máximo es de $132,3 \mathrm{mg} / \mathrm{Nm}^{3}$ que se presentó en el cuarto día, evidenciando que los datos se encuentran dentro de los límites máximos permisibles adicionalmente se demuestra que gran parte de los datos se encuentran fluctuando en el promedio de la media de los datos que es $9,6 \mathrm{mg} / \mathrm{Nm} 3$ y su valor mínimo $0,0 \mathrm{mg} / \mathrm{Nm}^{3}$.

\section{Variación diaria del monitoreo del dióxido de azufre (SO2) del Hospital General de Latacunga.}

En el presente gráfico se muestra la variación, del máximo, la media y el minino de los valores de concentración del dióxido de azufre obtenidos durante cinco de monitoreo.

Gráfico 2 Análisis diario del monitoreo del $\left(\mathrm{SO}_{2}\right)$.

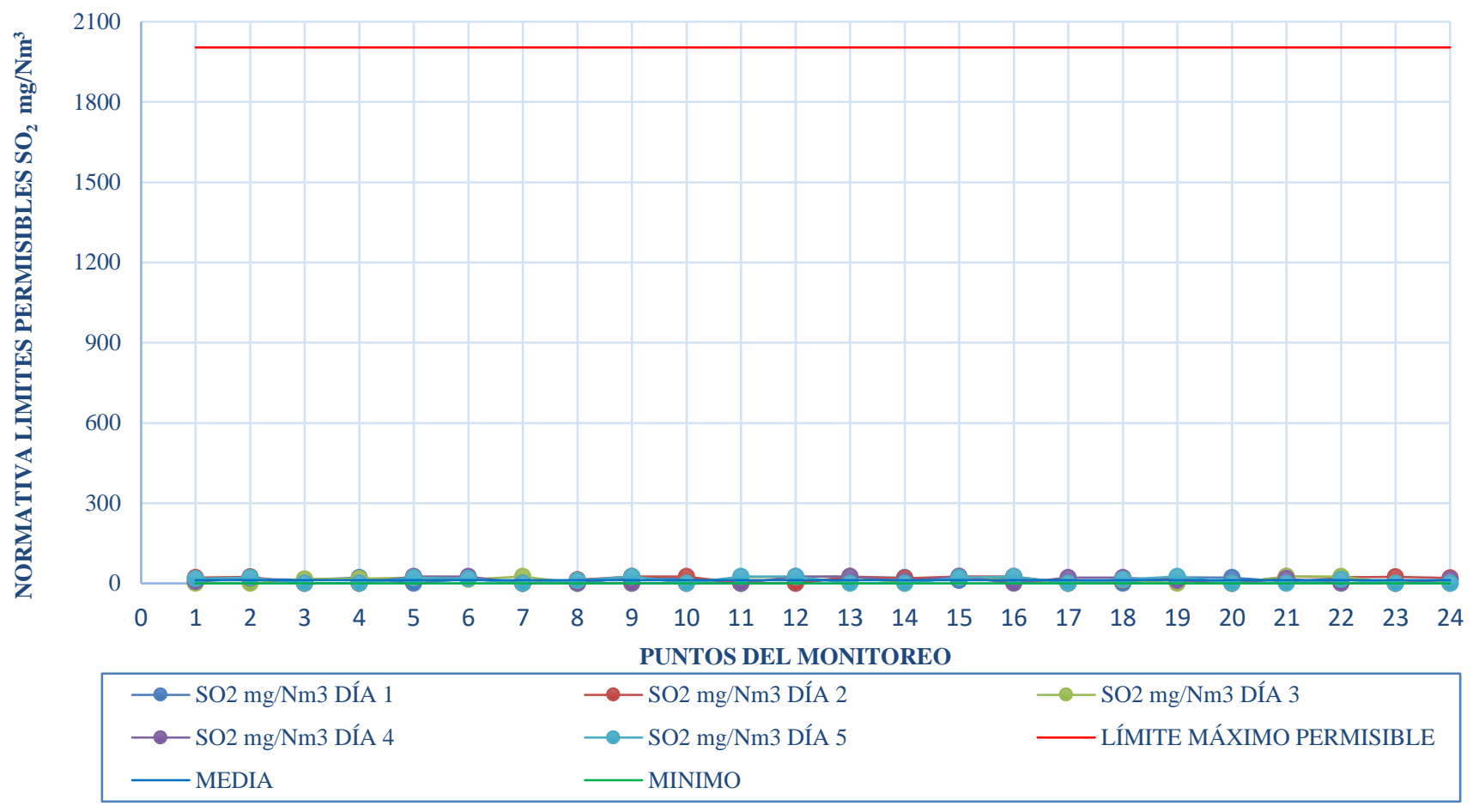

Autor: Grupo de Investigación.

\section{Interpretación del gráfico:}

En el gráfico se puede evidenciar los resultados del monitoreo realizado durante cinco días en sus 24 puntos de medición, donde se observa que el valor máximo es de $26 \mathrm{mg} / \mathrm{Nm}^{3}$ la 
cual se presentó en el día dos, tres, cuatro y quinto, evidenciando que los datos se encuentran dentro de los límites máximos permisibles adicionalmente se demuestra que gran parte de los datos se encuentran fluctuando en el promedio de la media de los datos que es 12,002 y su valor mínimo siendo $0,0 \mathrm{mg} / \mathrm{Nm}^{3}$.

\section{Resultados y análisis de los gases identificados en la Empresa de Cereales La Pradera.}

En la tabla se presentan los resultados de las emisiones de los gases monitoreados y su comparación con la normativa ambiental.

Tabla 8 Comparación de datos con el TULSMA Libro VI, Anexo 3 de la Empresa Cereales la Pradera: Caldero 1

EMPRESA CEREALES LA PRADERA: CALDERO 1

\begin{tabular}{|c|c|c|c|c|c|}
\hline GASES & UNIDADES & $\begin{array}{l}\text { PROMEDIO } \\
\text { DATOS } \\
\text { MEDIDOS }\end{array}$ & $\begin{array}{l}\text { NORMATIVA ACTUAL } \\
\text { REGISTRO } N^{\circ} 387 \text { DEL } \\
\text { TULSMA } \\
4 \text { DE NOVIEMBRE - } \\
2015\end{array}$ & $\begin{array}{l}\text { NORMATIVA } \\
\text { DEROGADA } \\
\text { REGISTRO } \\
\mathbf{N}^{\circ \quad \text { DEL }} \\
\text { TULSMA } \\
2013\end{array}$ & $\begin{array}{l}\text { CUMPLIMIENTO } \\
\text { DE LA } \\
\text { NORMATIVA }\end{array}$ \\
\hline TH & ${ }^{\circ} \mathrm{C}$ & 149,273 & - & - & NO APLICA \\
\hline $\mathbf{O}_{2}$ & $\%$ & 12,977 & - & - & NO APLICA \\
\hline $\mathrm{CO}_{2}$ & $\%$ & 59,114 & - & - & NO APLICA \\
\hline CO & $\mathrm{mg} / \mathrm{Nm}^{3}$ & 278,814 & - & 1800 & NO APLICA \\
\hline NOx & $\mathrm{mg} / \mathrm{Nm}^{3}$ & 121,636 & 670 & - & CUMPLE \\
\hline $\mathrm{SO}_{2}$ & $\mathrm{mg} / \mathrm{Nm}^{3}$ & 13,442 & 2004 & - & CUMPLE \\
\hline
\end{tabular}

Autor: Grupo de Investigación.

\section{Interpretación.}

Desarrollado el monitoreo de gases en fuentes fijas, teniendo como característica que el caldero es de vapor del año 2011 fuente fija existente: entrar en funcionamiento con la autorización del Libro VI, Anexo 3 del TULSMA emitido en el 2003. Se obtuvo como resultado en el caldero uno los siguientes valores: 121,636 mg/ $\mathrm{Nm}^{3} \quad \mathrm{NO}_{\mathrm{X}}$ (óxido de nitrógeno) y de $\mathrm{SO}_{2}$ (dióxido de azufre) 13,442 $\mathrm{mg} / \mathrm{Nm}^{3}$. Los mismos que fueron comparados con los valores: $670 \mathrm{mg} / \mathrm{Nm}^{3} \mathrm{NOx}$ y $2004 \mathrm{mg} / \mathrm{Nm}^{3} \mathrm{SO}_{2}$ de los gases respectivamente expuestos en TABLA 2 LIMITES MÁXIMOS PERMISIBLES DE CONCENTRACIÓN DE EMISIÓN DE CONTAMINANTES AL AIRE PARA CALDERAS. De tal manera queda demostrando que los valores de las concentraciones de los gases se encuentran dentro de los límites máximos permisibles. 
Análisis: En la Empresa Cereales La Pradera en su segundo caldero el parámetro Monóxido de carbono $\mathrm{CO}$ da como resultado: $278,814 \mathrm{mg} / \mathrm{Nm}^{3}$ pero en la normativa ambiental actual no aplica, mientras que en la Normativa derogada TULSMA del 2013 obedece a los límites máximos permisibles.

\section{Variación diaria del monitoreo del óxido de nitrógeno (NOx) de la Empresa de Cereales La Pradera de su primer caldero.}

En el presente grafico se muestra la variación, del máximo, la media y el minino de los valores de concentración de óxido de nitrógeno obtenidos durante cinco días de monitoreo.

\section{Gráfico 3 Análisis diario del monitoreo del (NOx)}

Autor: Grupo de Investigación.

\section{Interpretación del gráfico:}

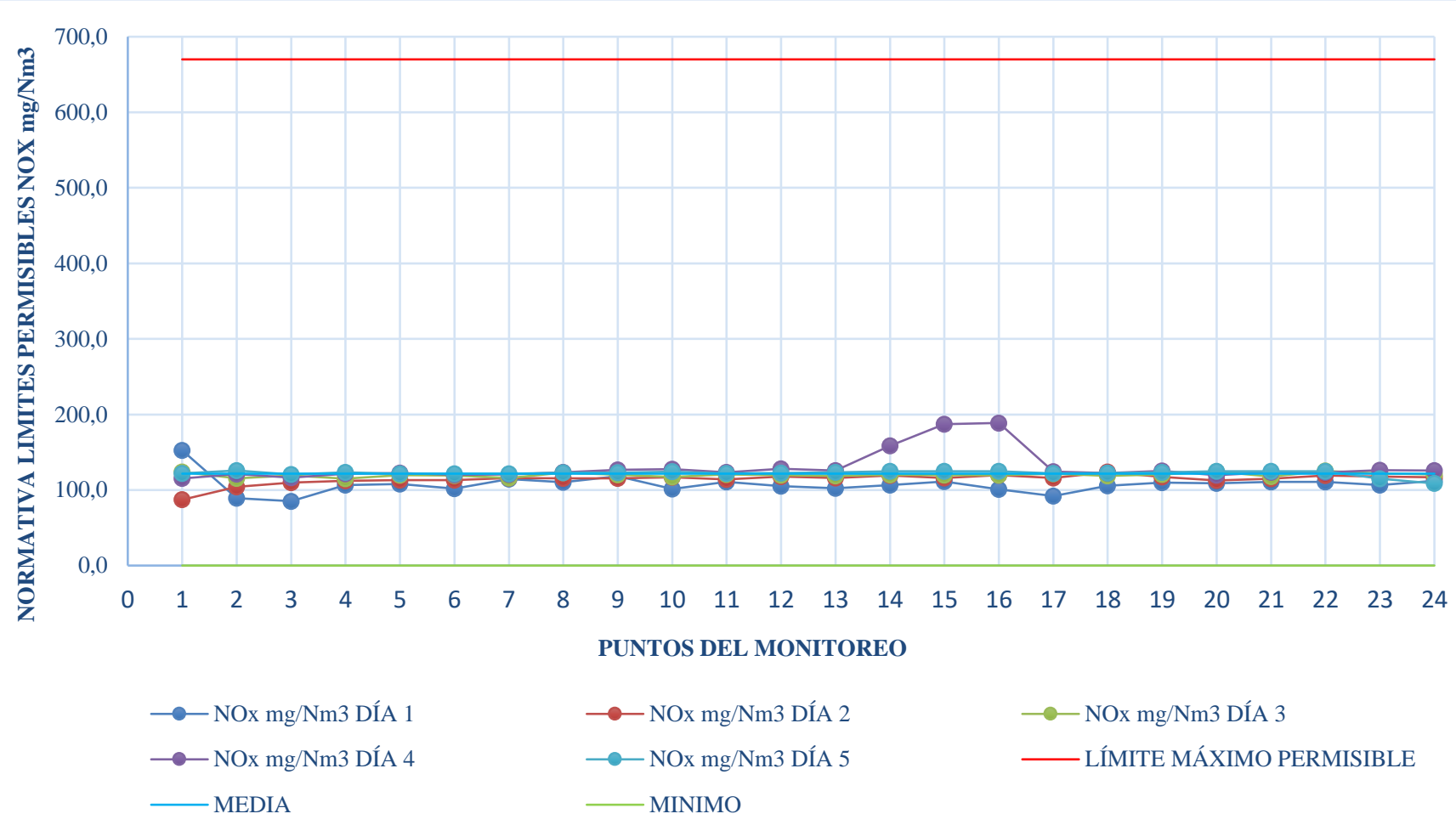

En el gráfico se puede evidenciar los resultados del monitoreo realizado durante cinco días en sus 24 puntos de medición, donde se observa que el valor máximo es de $188,8 \mathrm{mg} / \mathrm{Nm}^{3}$ que se presentó en el cuarto día, evidenciando que los datos se encuentran dentro de los límites máximos permisibles adicionalmente se demuestra que gran parte de los datos se encuentran fluctuando en el promedio de la media de los datos que es $121,6 \mathrm{mg} / \mathrm{Nm} 3 \mathrm{y}$ su valor mínimo $0,0 \mathrm{mg} / \mathrm{Nm}^{3}$. 
Variación diaria del monitoreo del dióxido de azufre $\left(\mathrm{SO}_{2}\right)$ de la Empresa de Cereales La Pradera de su primer caldero.

En el presente gráfico se muestra la variación, del máximo, la media y el minino de los valores de concentración de dióxido de azufre obtenidos durante cinco días de monitoreo.

Gráfico 4 Análisis diario del monitoreo del $\left(\mathrm{SO}_{2}\right)$

Autor: Grupo de Investigación.

Interpretación del gráfico:

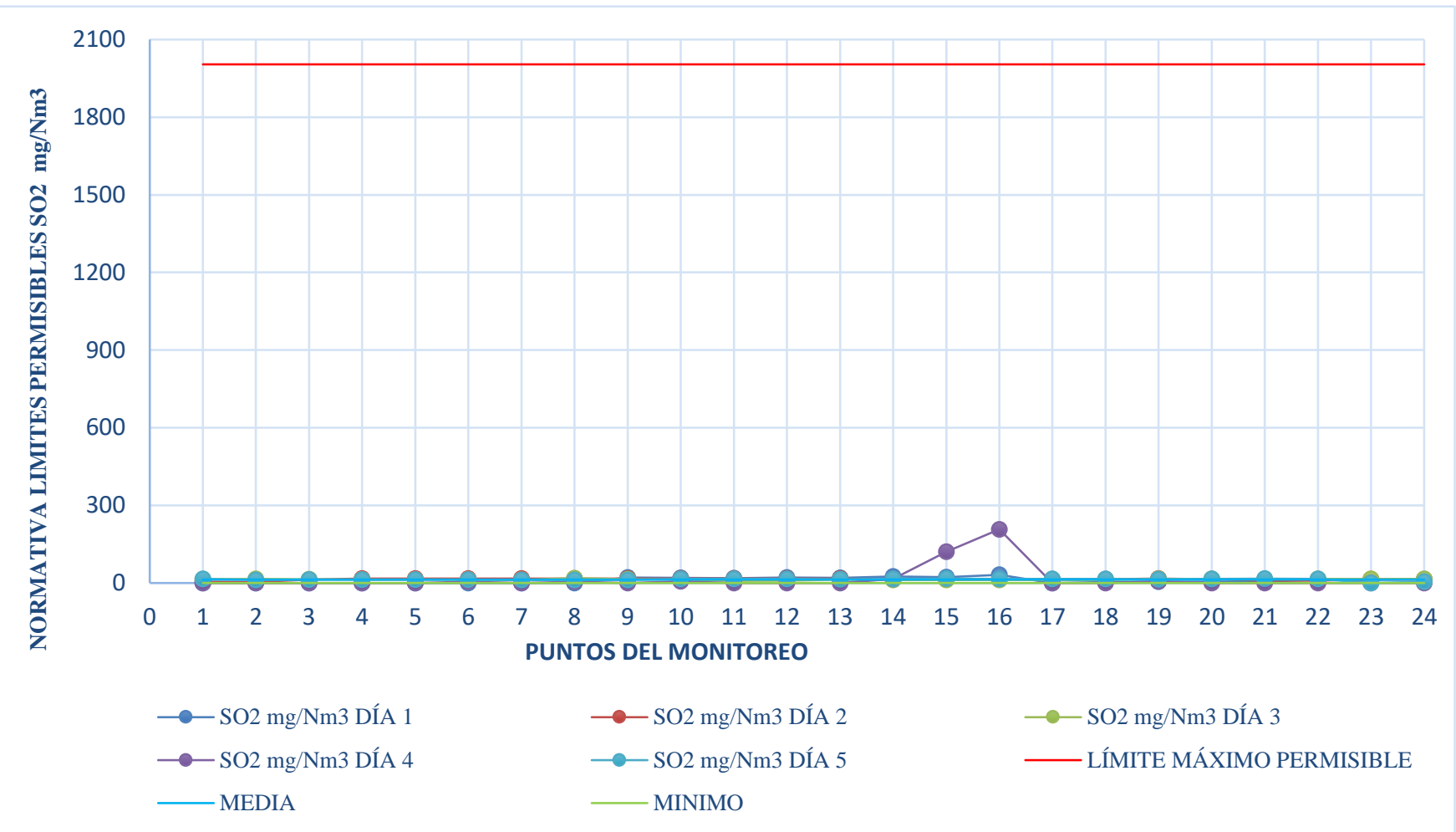

En el gráfico se puede evidenciar que los valores del dióxido de azufre $\left(\mathrm{SO}_{2}\right)$ en la Empresa En el gráfico se puede evidenciar los resultados del monitoreo realizado durante cinco días en sus 24 puntos de monitoreo, donde se observa que el valor máximo es de $208 \mathrm{mg} / \mathrm{Nm}^{3}$ que se presentó en el cuarto día, evidenciando que los datos se encuentran dentro de los límites máximos permisibles adicionalmente se demuestra que gran parte de los datos se encuentran fluctuando en el promedio de la media de los datos que es $13,4 \mathrm{mg} / \mathrm{Nm}^{3}$ y su valor mínimo $0,0 \mathrm{mg} / \mathrm{Nm}^{3}$. 
En la tabla se presentan los resultados de las emisiones de los gases monitoreados y su comparación con la normativa ambiental de la Empresa Cereales La pradera en su segundo caldero.

Tabla 9 Comparación con el TULSMA Libro VI, Anexo 3 de la Empresa Cereales la Pradera: Caldero 2

\begin{tabular}{|c|c|c|c|c|c|}
\hline GASES & UNIDADES & $\begin{array}{l}\text { PROMEDIOS } \\
\text { DATOS } \\
\text { MEDIDOS }\end{array}$ & $\begin{array}{l}\text { REGISTRO } \mathbf{N}^{\circ} 387 \text { DEL } \\
\text { TULSMA } \\
\text { REGISTRO } \mathbf{N}^{\circ} 387 \text { DEL } \\
\text { TULSMA } \\
4 \text { DE NOVIEMBRE - } 2015\end{array}$ & $\begin{array}{l}\text { NORMATIV } \\
\text { A } \\
\text { DEROGADA } \\
\text { REGISTRO } \\
N^{\circ} \quad \text { DEL } \\
\text { TULSMA } \\
2013\end{array}$ & $\begin{array}{l}\text { CUMPLIMIENTO } \\
\text { DE LA } \\
\text { NORMATIVA }\end{array}$ \\
\hline TH & ${ }^{\circ} \mathrm{C}$ & 129,581 & - & - & NO APLICA \\
\hline $\mathbf{O}_{2}$ & $\%$ & 13,310 & - & - & NO APLICA \\
\hline $\mathrm{CO}_{2}$ & $\%$ & 5,753 & - & - & NO APLICA \\
\hline $\mathrm{CO}$ & $\mathrm{mg} / \mathrm{Nm}^{3}$ & 161,816 & - & 1800 & NO APLICA \\
\hline NOx $_{\mathbf{x}}$ & $\mathrm{mg} / \mathrm{Nm}^{3}$ & 120,957 & 670 & - & CUMPLE \\
\hline $\mathrm{SO}_{2}$ & $\mathrm{mg} / \mathrm{Nm}^{3}$ & 44,563 & 2004 & - & CUMPLE \\
\hline
\end{tabular}

Autor: Grupo de Investigación.

\section{Interpretación del gráfico:}

Desarrollado el monitoreo de gases en fuentes fijas, teniendo como característica que el caldero es de vapor del año 2011 fuente fija existente: entrar en funcionamiento con la autorización del Libro VI, Anexo 3 del TULSMA emitido en el 2003. Se obtuvo como resultado en el caldero los siguientes valores: $120,957 \mathrm{mg} / \mathrm{Nm}^{3} \mathrm{NO}_{\mathrm{x}}$ (óxido de nitrógeno) y 44,563 mg/ $\mathrm{Nm}^{3} \mathrm{SO}_{2}$ (dióxido de azufre). Los mismos que fueron comparados con los valores: $\mathrm{NO}_{\mathrm{X}} 670 \mathrm{mg} / \mathrm{Nm}^{3}$ y SO $2004 \mathrm{mg} / \mathrm{Nm}^{3}$ de los gases respectivamente expuestos en TABLA 2 LIMITES MÁXIMOS PERMISIBLES DE CONCENTRACIÓN DE EMISIÓN DE CONTAMINANTES AL AIRE PARA CALDERAS. De tal manera queda demostrando que los valores de las concentraciones de los gases se encuentran dentro de los límites máximos permisibles.

Análisis: En la Empresa Cereales La Pradera en su segundo caldero el parámetro Monóxido de carbono $\mathrm{CO}$ da como resultado: $161,816 \mathrm{mg} / \mathrm{Nm}^{3}$ pero en la normativa ambiental actual no aplica, mientras que en la Normativa derogada TULSMA del 2013 obedece a los límites máximos permisibles. 
Variación diaria del monitoreo del óxido de nitrógeno (NOX) de la Empresa de Cereales La Pradera de su segundo caldero.

En el presente grafico se muestra la variación, del máximo, la media y el minino de los valores de concentración de óxido de nitrógeno obtenidos durante cinco días de monitoreo.

Gráfico 5 Análisis diario del monitoreo del $(\mathrm{NO})$

Autor: Grupo de Investigación.

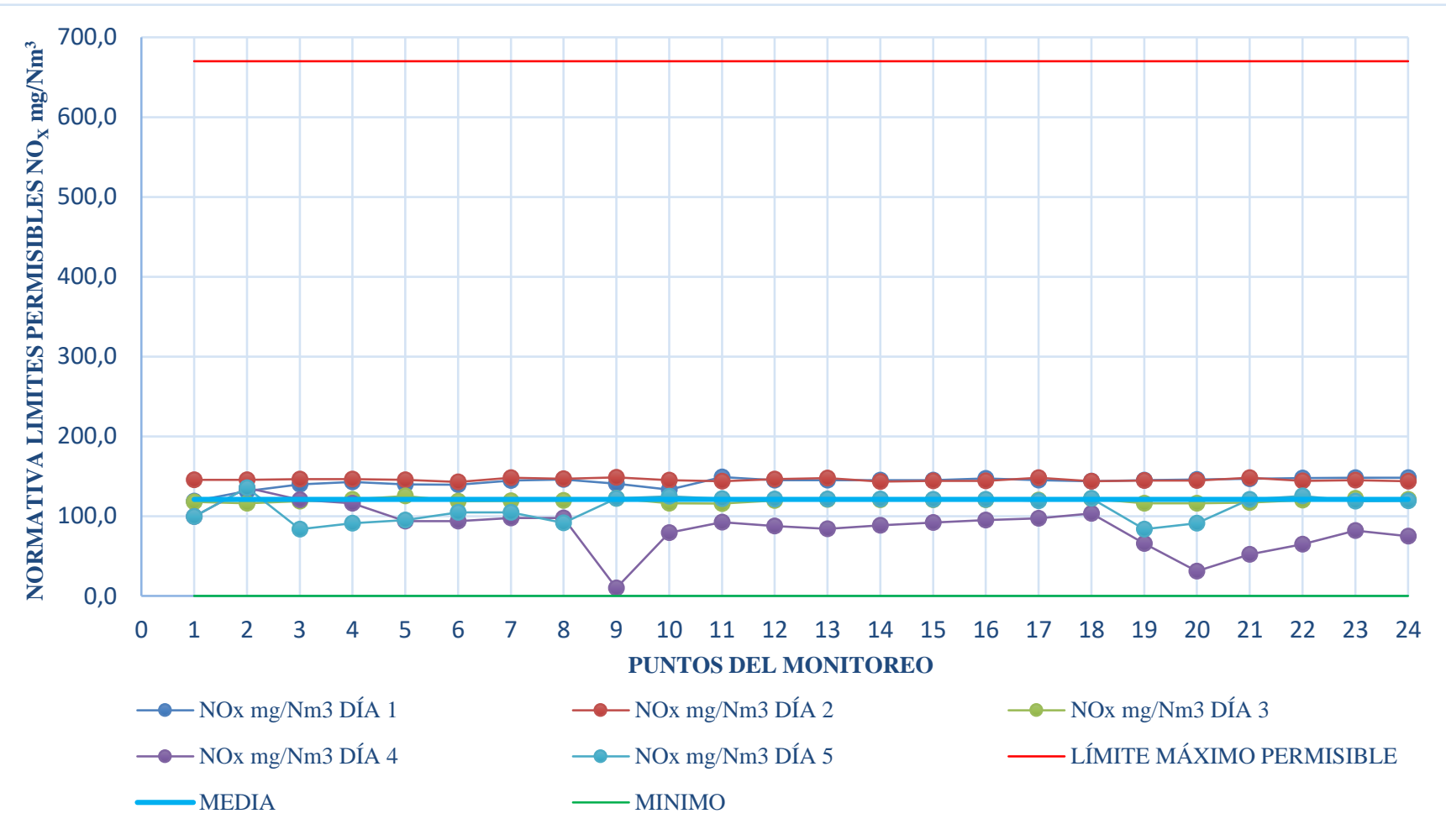

\section{Interpretación del gráfico:}

En el gráfico se puede evidenciar los resultados del monitoreo realizado durante cinco días en sus 24 puntos de monitoreo, donde se observa que el valor máximo es de $148,5 \mathrm{mg} / \mathrm{Nm}^{3}$ que se presentó en los día 2 y 4, evidenciando que los datos se encuentran dentro de los límites máximos permisibles adicionalmente se demuestra que gran parte de los datos se encuentran fluctuando en el promedio de la media de los datos que es $121 \mathrm{mg} / \mathrm{Nm}^{3}$ y su valor mínimo $0,0 \mathrm{mg} / \mathrm{Nm}^{3}$. 
Variación diaria del monitoreo en la Empresa de Cereales La Pradera Caldero 2 En el presente grafico se muestra la variación, del máximo, la media y el minino de los valores de concentración de dióxido de azufre obtenidos durante los 5 días de monitoreo.

Gráfico 6 Análisis diario del monitoreo del $\left(\mathrm{SO}_{2}\right)$

Autor: Grupo de Investigación.

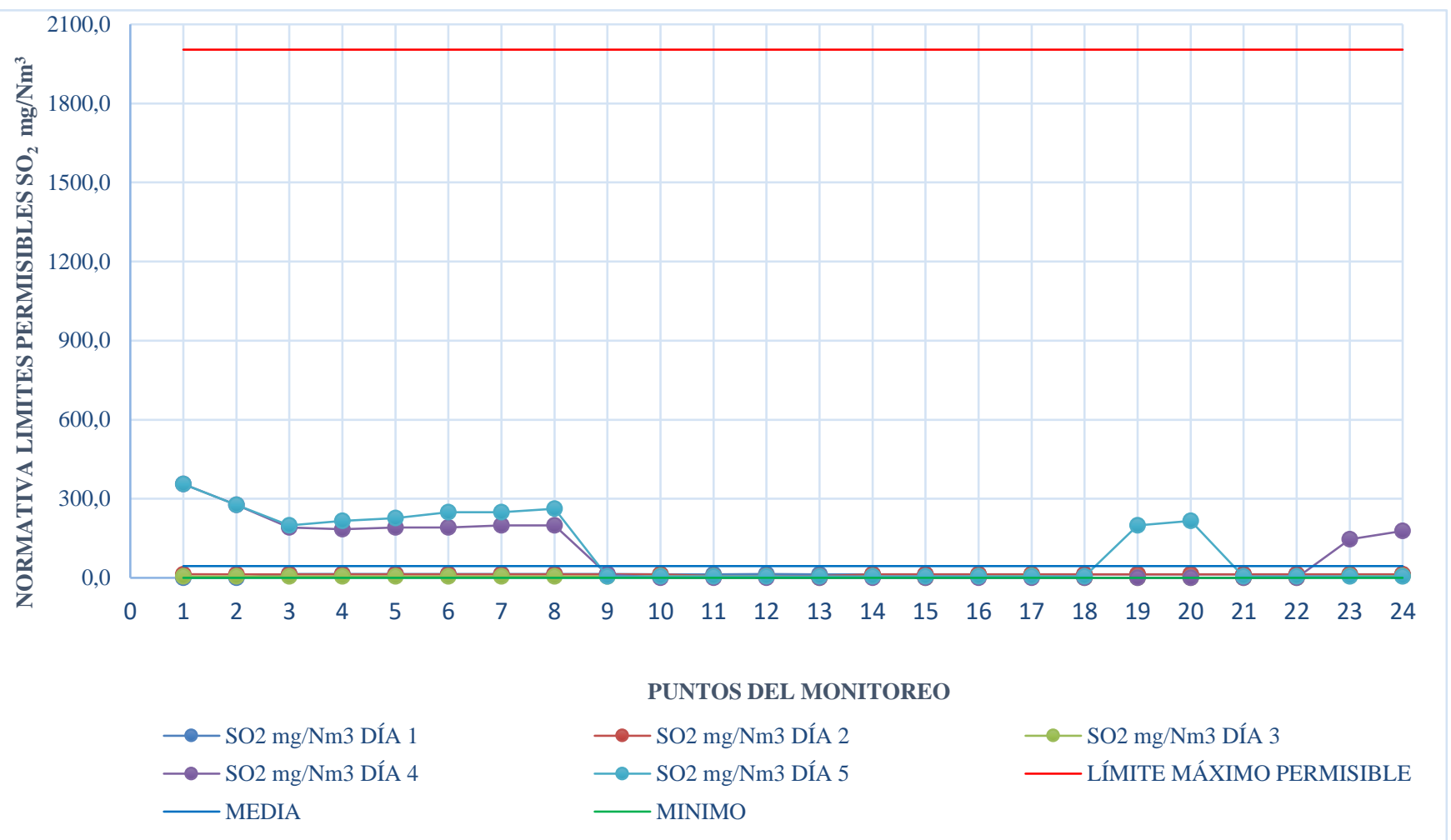

\section{Interpretación del gráfico.}

En el gráfico se puede evidenciar los resultados del monitoreo realizado durante los 5 días en sus 24 puntos de monitoreo, donde se observa que el valor máximo es de $356 \mathrm{mg} / \mathrm{Nm}^{3}$ que se presentó la cual se presentó en el día 5, evidenciando que los datos se encuentran dentro de los límites máximos permisibles adicionalmente se demuestra que gran parte de los datos se encuentran fluctuando en el promedio de la media de los datos que es $44,6 \mathrm{mg} / \mathrm{Nm}^{3}$ y su valor mínimo $0,0 \mathrm{mg} / \mathrm{Nm}^{3}$.

\section{Conclusiones.}

- Los monitoreos se llevaron acorde a lo establecido en la normativa ambiental vigente TULSMA LIBRO VI ANEXO 3 TABLA 2 y su aplicación de metodologías para la obtención de los datos de concentración de los gases contaminantes en fuentes fijas de combustión. 
- El Hospital General de Latacunga posee un caldero de vapor que trabaja desde el 2013 su funcionamiento es de lunes a domingo el cual es utilizado para áreas de esterilización y distribución de agua caliente a toda la planta durante 12 horas diarias depende la producción; presenta condiciones estructurales inadecuadas como: puertos de muestreo, escaleras, plataformas de trabajo para poder realizar una correcta medición.

- La Empresa Cereales La Pradera posee dos calderos que funciona desde el 2011 su funcionamiento es de lunes a sábado el cual es utilizado básicamente para calentar agua o generar vapor utilizado en la esterilización y distribución de agua caliente a toda la planta durante 6 horas diarias; presenta condiciones estructurales adecuadas como: puertos de muestreo, escaleras, plataformas de trabajo donde se realizó una correcta medición.

- Los resultados del monitoreo en el Hospital General de Latacunga fueron: 89,325 $\mathrm{mg} / \mathrm{Nm}^{3} \mathrm{NO}_{\mathrm{X}}$ y $12,002 \mathrm{mg} / \mathrm{Nm}^{3} \mathrm{SO}_{2}$. Los mismos que fueron comparados con los valores: $670 \mathrm{mg} / \mathrm{Nm}^{3}$ de $\mathrm{NO}_{x}$ y $2004 \mathrm{mg} / \mathrm{Nm}^{3}$ de $\mathrm{SO}_{2}$ de los gases respectivamente expuestos en TABLA 2 LIMITES MÁXIMOS PERMISIBLES DE CONCENTRACIÓN DE EMISIÓN DE CONTAMINANTES AL AIRE PARA CALDERAS. De tal manera queda demostrado que los valores de las concentraciones de los gases $\mathrm{NO}_{x}$ y $\mathrm{SO}_{2}$, se encuentra dentro de los límites máximos permisibles.

- Los resultados la Empresa de Cereales la Pradera fueron: 121,636mg/ $\mathrm{Nm}^{3} \mathrm{NOx}$, $13,442 \mathrm{mg} / \mathrm{Nm}^{3} \mathrm{SO}_{2}, 120,957 \mathrm{mg} / \mathrm{Nm}^{3} \mathrm{NOx}$ y $44,563 \mathrm{mg} / \mathrm{Nm}^{3} \mathrm{SO}_{2}$ de la chimenea uno y dos respectivamente. Los mismos que fue comparados con los valores: 670 $\mathrm{mg} / \mathrm{N} \mathrm{m}^{3}$ de $\mathrm{NO}_{\mathrm{X}}$ y $2004 \mathrm{mg} / \mathrm{Nm}^{3}$ de $\mathrm{SO}_{2}$ expuestos en TABLA 2 LIMITES MÁXIMOS PERMISIBLES DE CONCENTRACIÓN DE EMISIÓN DE CONTAMINANTES AL AIRE PARA CALDERAS. De tal manera queda demostrado que los valores de las concentraciones de los gases $\mathrm{NO}_{\mathrm{X}}$ y $\mathrm{SO}_{2}$, se encuentra dentro de los límites máximos permisibles

\section{Referencias bibliográficas.}

Acuario, B. (2012). VYC Industrial: Caldera de vapor en la industria alimenticia . Obtenido de VYC Industrial: Caldera de vapor en la industria alimenticia : http://vycindustrial.com/caldera-vapor-industria-alimenticia/

Alexis Yassi \& Tomas Kjellstrom. (2012). Riesgos Ambientales Para la Salud. España: INSHT(Instituto Nacional de Seguridad e Higiene en el Trabajo).

Almeida, A. A. (2010). INVENTARIO DE EMISIONES ATMOSFÉRICAS A PARTIR DE FUENTES FIJAS, MÓVILES Y DE ÁREA EN LA CIUDAD DE LATACUNGA. 
Obtenido de INVENTARIO DE EMISIONES ATMOSFÉRICAS A PARTIR DE FUENTES FIJAS, MÓVILES Y DE ÁREA EN LA CIUDAD DE LATACUNGA: http://www.dspace.uce.edu.ec/bitstream/25000/235/1/T-UCE-0012-37.PDF

Bahamondes, P. A. (03 de 04 de 2015). ACHS. Obtenido de http://www.achs.cl/qapub/trabajadores/Capacitacion/CentrodeFichas/Documents/des cripcion-de-caldera-y-generadores-de-vapor.pdf

Bonilla, Arranza, y Beltheton, A. (2009). Termodinámica de Equipos Industriales. Eficacia Energética de una Caldera., 18-34.

Bravo, H. Sosa, R. (12 de Abril de 2009). Caracteristicas de los Contaminantes Atmosféricos. Obtenido de Caracteristicas de los Contaminantes Atmosféricos: http://www.bvsde.paho.org/bvsacd/eco/036993-I/036993-1.1.pdf

Cazares Lusiana. (2012). Física y Química. Madrid: Editex, S.A.

CENTRO DE INFORMACIÓN DE LA CONTAMINACIÓN DEL AIRE, C. (199). Técnicas para calcular emisiones de categorías de fuentes únicas en su género en Mexicali. Mexicali: US EPA3. Obtenido de Técnicas para camcular emisiones de categorías de fuentes únicas en su género en MEXICALI.

Centro de Investigacion de Medio Ambiente . (21 de Febrero de 2012). Calidad del aire de Cantabria Consejeria de Medio Ambiente Ordenacion del territorio y urbanismo Gobierno de Cantabria. Obtenido de Calidad del aire de Cantabria Consejeria de Medio Ambiente Ordenacion del territorio y urbanismo Gobierno de Cantabria: https://www.airecantabria.com/CIAcontaminantes.php

Chile, C. N. (2009). GUÍA METODOLÓGICA PARA LA ESTIMACIÓN DE EMISIONES ATMOSFÉRICAS DE FUENTES FIJAS. Santiago de Chile: Departamento de Control de la Contaminación de CONAMA.

Córdova, P. (17 de 01 de 2013). Lección de Ciencia Naturales. Recuperado el 13 de 05 de 2017, de https://sites.google.com/site/lecciondeciencianaturales/el-calentamientoglobal/contaminacion-en-las-regiones-de-ecuador

Dinesen, C. (2010). Efectos del dióxido de carbono en la contaminación del aire. Recuperado el 04 de 05 de 2017, de http://www.ehowenespanol.com/efectos-deldioxido-carbono-contaminacion-del-aire-lista_444306 
Domínguez, J. (2008). Riesgos Laborales Relacionados con el Medio Ambiente. $\begin{array}{lllllll}\text { Recuperado el } & 15 & \text { de } & 05 & \text { de } & 2017, & \text { de }\end{array}$ http://www.inpahu.edu.co/biblioteca/imagenes/libros/Riesgos.pdf

Eduardo Mantilla, Crisanto Vergel \& Jose López. (2005). Medición de la Sostenibilidad Ambiental. Medellín: Universidad Cooperativa de Colombia.

Enciclopedia de Características. (2017). 10 Características de la Contaminación Atmosférica. Recuperado el 01 de 07 de 2017, de https://www.caracteristicas.co/contaminacion-atmosferica/

Eulalia, R. T. (2013). "DETERMINACIÓN DE LAS CONCENTRACIONES DE GASES DE COMBUSTIÓN DE FUENTES FIJAS DEL HOSPITAL GENERAL LATACUNGA Y EL HOSPITAL DE SEGURO SOCIAL DE LA CIUDAD DE LATACUNGA, PROVINCIA DE COTOPAXI PERIODO 2013". Obtenido de "DETERMINACIÓN DE LAS CONCENTRACIONES DE GASES DE COMBUSTIÓN DE FUENTES FIJAS DEL HOSPITAL GENERAL LATACUNGA Y EL HOSPITAL DE SEGURO SOCIAL DE LA CIUDAD DE LATACUNGA, PROVINCIA DE COTOPAXI PERIODO 2013": http://repositorio.utc.edu.ec/bitstream/27000/2754/1/T-UTC-00291.pdf

Fabiani, N. et al. (1999). Evolution of air pollution and impact of control programs in three Megacities of Latin America. En N. M.-G. Fabiani, Evolution of air pollution and impact of control programs in three Megacities of Latin America. (págs. 203-215). Mexico.

Gallego, A. et al., . (2012). Contaminación Atmosférica: De la Universidad Nacional de Educacion a Distancia Madrid 2012. Madrid: Edición digital. Obtenido de Gallego, A., Gonzales I., Sanchez, B., Fernandez, P.,Garcinuño, R., Bravo,J., Pradana., J. (2004) Contaminación Atmosférica: Edición digital: noviembre de 2012 de la Universidad Nacional de Educacion a Distancia Madrid 2012.

Gallego, et al. (2012). contaminación atmosférica. Madrid: Edicion Digital.

Geographic, N. (5 de Diciembre de 2013). La contaminación del aire -medio - ambiente. Obtenido de La contaminación del aire -medio - ambiente: http://www.nationalgeographic.es/medio-ambiente/la-contaminacion-del-aire

Guerrero, D. d., Ibarra, A., \& Fausto, I. (2004). Ciencia. España: Complutensa. 
Hernández, M. (21 de Abril de 2010). Plan Nacional de la Calidad del Aire . Obtenido de Plan Nacional de la Calidad del Aire : http://www.ambiente.gob.ec/wpcontent/uploads/downloads/2012/10/libro-calidad-aire-1-final.pdf

Keller, E., Blodgett, R. (2007). Riesgos Naturales: Procesos de la Tierra como Riesgos, desastres y catástrofes. Madrid: Pearson Educación, S.A.

MAE. (18 de Enero de 2012). Sistema de Contabilidad Ambiental Nacional Exploración Inicial. Obtenido de Sistema de Contabilidad Ambiental Nacional Exploración Inicial: http://suia.ambiente.gob.ec/documents/10179/242984/6.+Cuenta+de+Emisiones+al+ Aire.pdf/9ffc6202-2c7d-4bef-b3be-

8133da96dd78;jsessionid=RZBfIKdnP5F0Sq5dm7t9yOS3?version=1.0

MAE. (5 de Junio de 2014). Preocupante situación ambiental en el Ecuador. Obtenido de Preocupante situación ambiental en el Ecuador: http://www.ambiente.gob.ec/wpcontent/uploads/downloads/2012/08/Planes-y-Programas-de-ejecucion2012_Agosto.pdf

Mantilla, E., Vergal, C., \& López, J. (2005). Medición de la Sostenibilidad Ambiental. Bogotá: Universidad Cooperativa de Colombia (Educc).

Manual del Testo 350. (2008). Global Instrumental. Recuperado el 2017, de https://www.testo.com.ar/resources/media/global_media/produkte/testo_350/Testo_3 50_-_Manual.pdf

Manual Testo 350. (2015). Obtenido de Empresa líder en la distribución de equipos de climatización. Obtenido de Obtenido de Empresa líder en la distribución de equipos de climatización: http://www.anwo.cl/testo-350---completo-analizador-de-gasespara-la-gran-industria/ficha.html?p=17000\&c=2024\&u=3

María Nieves Gonzalez Delgado, C. O. (2011). Contaminación ambiental: una visión desde la química. Eapaña: Ediciones Paraninfo S.A.

Martínez, E., \& Díaz de Mera, M. (2004). Contaminación Atmosférica. Cuenca: Ediciones de la Universidad de Castilla- La Mancha.

Ministerio de Agricultura y Pesca, Alimentación y Medio Ambiente. (2007). SOX (ÓXIDOS DE AZUFRE). Obtenido de http://www.prtr-es.es/SOx-oxidos-deazufre, 15598,11,2007.html 
Ministerio del Ambiente . (2010). Plan Nacional de Calidad del Aire. Recuperado el 14 de 05 de 2017, de http://www.ambiente.gob.ec/wpcontent/uploads/downloads/2012/10/libro-calidad-aire-1-final.pdf

Ministerio del Ambiente . (2010). Plan Nacional de Calidad del Aire. Recuperado el 14 de 05 de 2017, de http://www.ambiente.gob.ec/wpcontent/uploads/downloads/2012/10/libro-calidad-aire-1-final.pdf

Ministerio del Ambiente. (13 de Febrero de 2014). Inventario Preliminar de las Emisiones de Contaminantes del Aire, de los cantones Ambato,Riobamba, Santo Domingo de los Colorados,Latacunga, Ibarra, Manta, Portoviejo, Esmeraldas y Milagro. Obtenido de Inventario Preliminar de las Emisiones de Contaminantes del Aire, de los cantones Ambato,Riobamba, Santo Domingo de los Colorados,Latacunga, Ibarra, Manta, Portoviejo, Esmeraldas y Milagro: http://www.ambiente.gob.ec/wpcontent/uploads/downloads/2014/05/Libro-Resumen-Inventario-13-02-2014prensa.pdf

Moreira, A. (11 de 03 de 2015). Ecured Conociemiento con todos y para todos . Obtenido de Ecured Conociemiento con todos $\mathrm{y}$ para todos : https://www.ecured.cu/Contaminantes_antropog\%C3\%A9nicos

Muerza Belmonte, R. (27 de 10 de 2010). Obtenido de http://mayores.uji.es/blogs/antropvinaros/2012/03/16/chimeneas-industriales-deladrillo/

Muerza, A. (2010). Aerosoles atmosféricos, como afectan al clima y a la salud. Recuperado el 2017, de http://www.consumer.es/web/es/medio_ambiente/naturaleza/2010/10/21/196641.php

National Geographic. (2000). Medición y Análisis de Contaminantes del Aire. Recuperado el 2017, de http://sisbib.unmsm.edu.pe/bibvirtualdata/libros/geologia/gestion_calidad/Cap08.pdf

OMS, P. d. (24 de Mayo de 2004). Asamblea de las Naciones Unidas sobre el Medio Ambiente del Programa de las Naciones Unidas para el Medio Ambiente. Obtenido de Asamblea de las Naciones Unidas sobre el Medio Ambiente del Programa de las Naciones Unidas para el Medio Ambiente: http://wedocs.unep.org/bitstream/handle/20.500.11822/17578/K1600805\%20Doc\%2 $06 \% 20$ S.pdf? sequence $=12 \&$ isAllowed $=y$ 
Organización Mundial de la Salud. (2014). La calidad del aire se está deteriorando en muchas de las cuidades del mundo. Obtenido de http://www.paho.org/arg\%20\%20/index.php?option=com_content\&view=article\&id $=1314$ :la-calidad-del-aire-se-esta-deteriorando-en-muchas-de-las-ciudades-delmundo\&catid=334: $\arg 04$-salud-ambiental-y-desarrollo-sustentable \&Itemid=228

Organización Mundial de la Salud. (2016). Calidad del aire ambiental (exterior) y salud. $\begin{array}{lllllll}\text { Recuperado el } & 18 & \text { de } & 04 & \text { de } & 2017, & \text { de }\end{array}$ http://www.who.int/mediacentre/news/releases/2016/air-pollution-estimates/es/

Orozco. (2003). Contaminación ambiental: Una visión desde la química. Madrid: Ediciones Paraninfo, SA.

Pérez, A. (2010). Ingeniería del Medio Ambiente. San Vicente: Club Univestario.

Pérez, S. (2014). Introducción a la Química y el Ambiente. . México: Grupo Editorial Patria.

Pérez, S. (2014). Introducción a la Química y el Ambiente. México: Grupo Editorial Patria.

Plan de Desarrollo y Ordenamiento Territorial. (20 de Enero de 2016). DIAGNOSTICO DEL PLAN DE DESARROLLO DEL CANTÓN LATACUNGA 2016-2019. Obtenido de DIAGNOSTICO DEL PLAN DE DESARROLLO DEL CANTÓN LATACUNGA 2016-2019: http://app.sni.gob.ec/snilink/sni/PORTAL_SNI/data_sigad_plus/sigadplusdocumentofinal/0560000380001_P DyOT\%20LATACUNGA\%201_20-04-2015_16-06-55.pdf

Ramírez, V. (2014). Química 2: Serie integral por competencias. México: Grupo Editorial Patria.

Red Ambiental de Asturias. (12 de Abril de 2013). Portal Medioambiental de Asturias: Conceptos generales de contaminación atmosférica. Obtenido de Portal Medioambiental de Asturias: Conceptos generales de contaminación atmosférica.: https://www.asturias.es/portal/site/medioambiente/menuitem.1340904a2df84e62fe47 $421 \mathrm{ca} 6108 \mathrm{a} 0 \mathrm{c} /$ ?vgnextoid=c92ad84189f32

Registro Estatal de Emisiones y Fuentes Contaminantes. (2007). CO (MONÓXIDO DE CARBONO). Recuperado el 11 de 04 de 2017, de http://www.prtr-es.es/COMonoxido-de-carbono, 15589,11,2007.html

Ricaute, J. (06 de 2013). http://www.mapama.gob.es/es/calidad-y-evaluacionambiental/temas/atmosfera-y-calidad-del-aire/emisiones/prob-amb/particulas.aspx. Obtenido de http://www.mapama.gob.es/es/calidad-y-evaluacion- 
ambiental/temas/atmosfera-y-calidad-del-aire/emisiones/prob-amb/particulas.aspx: http://www.mapama.gob.es/es/calidad-y-evaluacion-ambiental/temas/atmosfera-ycalidad-del-aire/emisiones/prob-amb/particulas.aspx

Rico, F., López, R., \& Figueroa, E. (2001). Daños a la salud por Contaminación Ambiental. México: Universidad Autónoma del Estado de México.

Rocha, L. (05 de 06 de 2014). Medio Ambiente: una ciudad con buenos aires, pero no tanto. La OMS coloca a la Capital entre las mejores de América latina. Recuperado el 01 de 06 de 2017, de http://www.estrucplan.com.ar/noticias/imprimirss.asp?IDNoticia=10720

Target Asesores S.A. (2016). Expertos en Gestión Medioambiental. España: IC Editorial.

Texto Unificado de Legislación Secundaria del Ministerio del Ambiente. (2017). Características de la Contaminación Atmosférica. Recuperado el 01 de 07 de 2017, de Características de la Contaminación Atmosférica: http://www.ambiente.gob.ec/wpcontent/uploads/downloads/2012/10/Acuerdo-50-NCA.pdf

TUlSMA. (2003). Norma De Emisiones Al Aire Desde Fuentes Fijas De Combustión. Libro VI, Anexo 3 Ecuador. Obtenido de Norma De Emisiones Al Aire Desde Fuentes Fijas De Combustión. Libro VI, Anexo 3 Ecuador: https://www.google.com.ec/url? sa=t\&rct=j\&q=\&esrc=s\&source=web\&cd=4\&cad=rj a\&uact=8\&ved=0ahUKEwiGo9fzsKPNAhVMHR4KHdygAhQQFggv

TULSMA, M. d. (2015). Norma de emisiones al aire desde fuentes fijas. Ecuador. Libro VI TULSMA Anexo III.

\section{LCiencia}




\section{Para citar el artículo indexado.}

Daza O., Clavijo M., Lozano C., Mantilla C. . (2018). Determinación de los gases contaminantes $\mathrm{O} 2, \mathrm{CO} 2, \mathrm{CO}$, NOX y SO2 en fuentes fijas en la provincia de Cotopaxi. Revista electrónica Ciencia Digital 2(4.2), 5-30. Recuperado desde: http://cienciadigital.org/revistacienciadigital2/index.php/CienciaDigital/article/view/199/17 $\underline{6}$

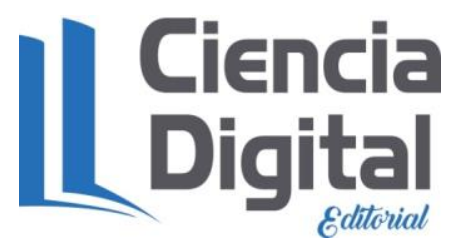

El artículo que se publica es de exclusiva responsabilidad de los autores y no necesariamente reflejan el pensamiento de la Revista Ciencia Digital.

El articulo queda en propiedad de la revista y, por tanto, su publicación parcial y/o total en otro medio tiene que ser autorizado por el director de la Revista Ciencia Digital.
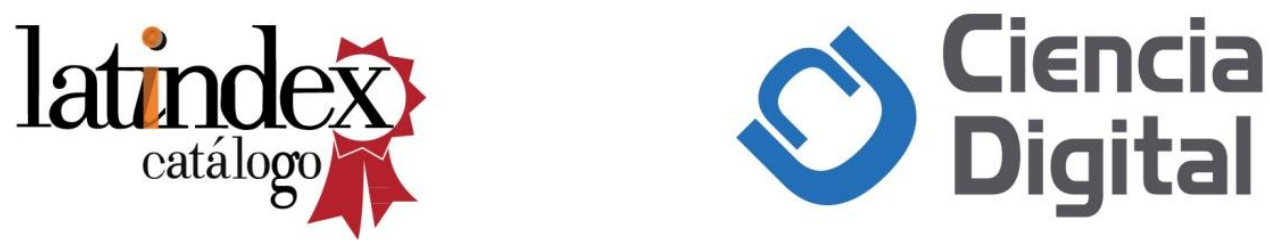